\title{
Cone-beam Computed Tomographic Evaluation of the Anatomical Changes of Temporomandibular Joint Use of Pre-post Dentures: A Time-control Study
}

\author{
Pankaj K Singh ${ }^{1}$, Nikita Raman ${ }^{2}$, Vaibhav K Singh ${ }^{3}$, Saifullah Akhtar ${ }^{4}$, Prabhat Mishra ${ }^{5}$, Sunny Sharma ${ }^{6}$
}

\begin{abstract}
Aim and objective: The growth of the temporomandibular joint (TMJ) gets affected by multiple factors like aging, occlusion state, and by the movement of the jaw while masticating and swallowing. Radiographic imaging is often utilized as a vital diagnostic adjunct in the evaluation of certain examinations of the TMJ.

Materials and methods: In this in vivo study, 30 male participants with mean age 55 years, having edentulous maxillary and mandibular arches from the Outpatient Department of Prosthodontics, were randomly selected. Group I $(n=30)$ patients who were edentulous for the last 4-5 years but without wearing dentures. Whereas group II $(n=30)$ patients who were edentulous for the last $4-5$ years but were wearing dentures for this period. Maxillary and mandibular dentures were fabricated and delivered to subjects. Subjects were subjected to the TMJ analysis with the help of CBCT. Radiological images of dentomaxillofacial structures were analyzed by a specialist with a dual monitor inside a darkened silent room. On the monitor, three times measurements were recorded followed by calculation of mean value. The recordings were taken on both sides and thus, 210 sites were analyzed altogether, followed by the statistical analysis using SPSS software version 15.0.

Results: The mean ages of group I and II were $59.00 \pm 6.74$ and $58.27 \pm 6.75$ years, respectively. The intra- and intergroup comparisons were done using a one-sample $t$-test. Differences in mean intercondylar width in groups I and II were not found to be statistically significant. The difference in mean length of glenoid fossa was not statistically significant at any of the above observation periods. A continuous decline in mean length of glenoid fossa was observed with time in both groups. The range of change in articular eminence length was found to be statistically significant for both the groups $(p<0.05)$.

Conclusion: This study shows that the articular eminence flattening is correlated with age; on the other hand, the rate of deformation was found significantly more in total edentulous subjects as compared to subjects having normally maintained occlusion. The anatomical changes inside the TMJ have been much greater expressed within the completely edentulous subjects in whom the angle of sagittal condyle path declines and so does the articular eminence height.

Clinical significance: It is essential to provide the edentulous patient with early prosthetic and occlusal rehabilitation after extractions to prevent the anatomical changes in TMJ.
\end{abstract}

Keywords: Articular eminence, Computed tomography, Glenoid fossa, Temporomandibular joint.

The Journal of Contemporary Dental Practice (2020): 10.5005/jp-journals-10024-2987

\section{INTRODUCTION}

Evaluation of the temporomandibular joint (TMJ) is important for the use of denture at the time of the edentulous period, which results in complicit esthetic and dietary habits. It also helped in the spread of awareness of using denture at the edentulous period as prolongation of edentulism leads to certain mandibular dysfunction/disease and results in the health of the patients. Previous morphological studies of the TMJ have established that age, occlusion state, and the movement of the jaw while masticating and swallowing have to influence on TMJ growth. ${ }^{1}$ The TMJ is ginglymoid arthrodial articulation with some degree of diarthrosis formed by the mandibular condyle and glenoid fossa of the temporal bone. ${ }^{2}$ During mandibular movements, the condylar process slides on the articular eminence of the temporal bone. The inclination of articular eminence differs between individuals and states the condylar movement path along with the extent of disc radiation over the condyle. ${ }^{3}$ Although it is an anatomical structure, but the morphological shape is influenced by functional load occurring from masticatory forces. ${ }^{4,5}$

Radiographic imaging is often utilized as a vital diagnostic adjunct in the orthodontic patient evaluation and intermittently includes certain specific examinations of the TMJ. Diagnostic
'Department of Prosthodontics Crown and Bridges and Implantology, Dental College Azamgarh, Uttar Pradesh, India

${ }^{2}$ Department of Dentistry, Anugrah Narayan Magadh Medical College and Hospital, Gaya, Bihar, India

${ }^{3}$ Department of Conservative, Dental College Azamgarh, Uttar Pradesh, India

${ }^{4}$ Department of Public Health Dentistry, Dental College Azamgarh, Uttar Pradesh, India

${ }^{5}$ Department of Prosthodontics Crown and Bridges and Implantology, Career Institute of Dental Sciences and Hospital, Lucknow, Uttar Pradesh, India

${ }^{6}$ Department of Prosthodontics Crown and Bridges and Implantology, Himachal Institute of Dental Sciences, Paonta Sahib, Himachal Pradesh, India

Corresponding Author: Nikita Raman, Department of Dentistry, Anugrah Narayan Magadh Medical College and Hospital, Gaya, Bihar, India, Phone: +91 9304518574, e-mail: nikitaraman07@gmail.com

How to cite this article: Singh PK, Raman N, Singh VK, et al. Conebeam Computed Tomographic Evaluation of the Anatomical Changes of Temporomandibular Joint Use of Pre-post Dentures: A Time-control Study. J Contemp Dent Pract 2020;21(12):1354-1360.

Source of support: Nil

Conflict of interest: None

() Jaypee Brothers Medical Publishers. 2020 Open Access This article is distributed under the terms of the Creative Commons Attribution 4.0 International License (https://creativecommons.org/licenses/by-nc/4.0/), which permits unrestricted use, distribution, and non-commercial reproduction in any medium, provided you give appropriate credit to the original author(s) and the source, provide a link to the Creative Commons license, and indicate if changes were made. The Creative Commons Public Domain Dedication waiver (http://creativecommons.org/publicdomain/zero/1.0/) applies to the data made available in this article, unless otherwise stated. 
methods for TMJ imaging have been considerably developed in recent decades like orthopantomography (OPG), computed tomography (CT) scan, magnetic resonance imaging (MRI), and cone-beam CT (CBCT). ${ }^{6-9}$

Cone-beam CT captures the area of interest with rapid scan time (10-70 seconds) minimizes the exposure of adjacent structures, while exposing the patient to low-radiation doses. ${ }^{10}$ The reconstruction of images in a 3D data set is done using a modified Feldkamp algorithm. Hence, data can be reformatted in volume rather than a slice, thereby giving 3D information. ${ }^{11-13}$ Cone-beam CT can provide high-quality diagnostic images with a submillimeter resolution, short scanning times, and lower radiation dose. Although the results are promising, still further research is required to assess the performance of different protocols for CBCT in TMJ imaging, especially in the detection of condylar defects. ${ }^{14,15}$

Several parameters make CBCT a better option than CT and MRI, like the dimension of the field of view (FOV), X-ray beam factors, the detector, and the dimension of the reconstructed voxels. ${ }^{16-18}$ A CBCT study would assay the morphological changes within the TMJ of a long-term edentulous patient who has been rehabilitated with complete denture. ${ }^{19}$

Hence, this present study aims to evaluate the relationship of the hard and soft tissue of TMJ in edentulous subjects before and after insertion of complete dentures using CBCT.

\section{Materials and Methods}

The present study was performed in the Department of Prosthodontics, Saraswati Dental College, Lucknow, Uttar Pradesh, India. The institutional ethical committee clearance was obtained (SDC/IHEC/2014/Thesis Pro forma/29) before starting the study. A total of 60 male subjects visiting the department from May 2015 to December 2016 with age group 49-75 years (mean age $=55$ years), having edentulous maxillary and mandibular arches from 4-5 years were randomly selected from the Outpatient Department of Prosthodontics of a private dental college in Lucknow, India, following the inclusion and exclusion criteria.

All the study subjects were informed about the nature of the research procedure in the language that they can understand, and a written consent was obtained. To standardize the selection of study subjects because of any skeletal aberrations, inclusion criteria were patients with healthy residual ridges showing Angle's Class I ridge relations and no history of pre-prosthetic surgery whereas patients with TMJ disorders facial asymmetries, history of uncontrolled systemic disorders or poor neuromuscular control, or highly resorbed ridges and poor mental attitude of the subjects were excluded.

Subjects were divided into two groups. Group I included patients who were edentulous from last 4-5 years but without wearing dentures. Whereas group II included patients who were edentulous from last 4-5 years but were wearing dentures for this period (till the study was not conducted) and were provided with a new set of the denture on the onset of the study. Reason for the new set of dentures for the patient who were wearing denture already was to evaluate the changes on the TMJ when the patient wears a new set of dentures that is the baseline of the study.

Subjects were taken to an imaging center for the TMJ analysis with the help of CBCT. Denture fabrication was done by conventional methodology. It is needed to be described as there is a various method of fabrication of denture and the choice of fabrication was conventional methodology as it is the primary method for denture fabrication. Primary impressions were made, and the primary cast was obtained. This was followed by fabrication of custom tray, and the final impression making obtaining master cast. Upon the master cast, maxillary and mandibular permanent denture bases, occlusal rims were fabricated and checked in the mouth for comfort, retention, stability, support, lip fullness, and support. Then, the anterior and posterior plane of occlusion was established concerning the interpupillary line and camper's plane, respectively. Vertical jaw relation and tentative centric relation were registered.

On a semi-adjustable Hanau articulator, maxillary cast was mounted with a face bow transfer and mounting of the mandibular cast was done in the centric relation. The mandibular occlusal rim was reduced $2 \mathrm{~mm}$ for the free movement of tracers followed by attachment of central bearing point of gothic arch tracer (Hanau Hight Tracers) about the lines drawn from the anterior border of the right and left retromolar pad of one side to the canine region of the contralateral side and then mandibular cast midline was drawn. The junction of these three lines was marked with an indelible pencil corresponding to the central bearing point. On the other hand, central bearing plates were attached to the maxillary occlusal rims. Extraoral tracers attached to the maxillary and mandibular occlusal rims were transferred to the subjects and extraoral tracings were obtained by asking the subjects to close at centric and do right lateral, left lateral, and protrusive movement and the tracer assembly was removed from the subject's mouth. A plastic sheet of $1 \mathrm{~mm}$ thickness was adapted over the extraoral tracer plate surface of the mandibular tracer assembly with sticky wax very carefully. A hole is made on the adapted plastic sheet of $1 \mathrm{~mm}$ thickness on the centric point of gothic arch tracing and the patient was guided to $C R$, ensuring that the needle part was in intimate contact with the hole at centric point recorded and kept for further comparison. Try-in was done, and later the patient was provided with a new set of a complete denture having centric relation with Angles Class I malocclusion and all necessary post-insertion instructions were given to them.

The CBCT images of the bilateral TMJ were obtained with a 3D Accuitomo CBCT machine (MCT-1 [EX-2F], Morita Manufacturing Corp, Kyoto, Japan) with image-capture parameters set at $85.0 \mathrm{kV}$ and $4.0 \mathrm{~mA}$ and an exposure time of 17.5 seconds. The voxel size was $0.125 \mathrm{~mm}$, and the slice thickness was $1.0 \mathrm{~mm}$. The FOV size was 120 $\mathrm{mm}$. Using Machine Imaging Science International (USA) I-CAT CB 500 , Software I-Cat Vision, axial, coronal, and sagittal 2D sectional images were assessed (Fig. 1). The images were assessed in a silent darkened room by a dentomaxillofacial radiology specialist with dual monitors (HPLP2475W, resolution $1920 \times 1200$ ). To ensure the best possible visualization, an image-processing tool was utilized in software for adjustments in brightness and contrast of the images. On the monitor, three times measurements were recorded followed by calculation of mean value by a single observer for intraexaminer reliability. The parameters recorded were intercondylar width, interarticular distance, length of the glenoid fossa, and articular eminence at different time intervals, i.e., at baseline, after $6,12,18$, and 24 months post-insertion. The data were recorded on both the sides for condyle, glenoid fossa, and articular eminence (60 patients were analyzed bilaterally for three parameters, thus making a total of 320 sites) and interarticular distance was analyzed for 60 patients, thus a total of 240 sites were checked $(320+60=380$ sites).

The data obtained were subjected to the statistical analysis using the SPSS version 15.0 software. The statistical significance 

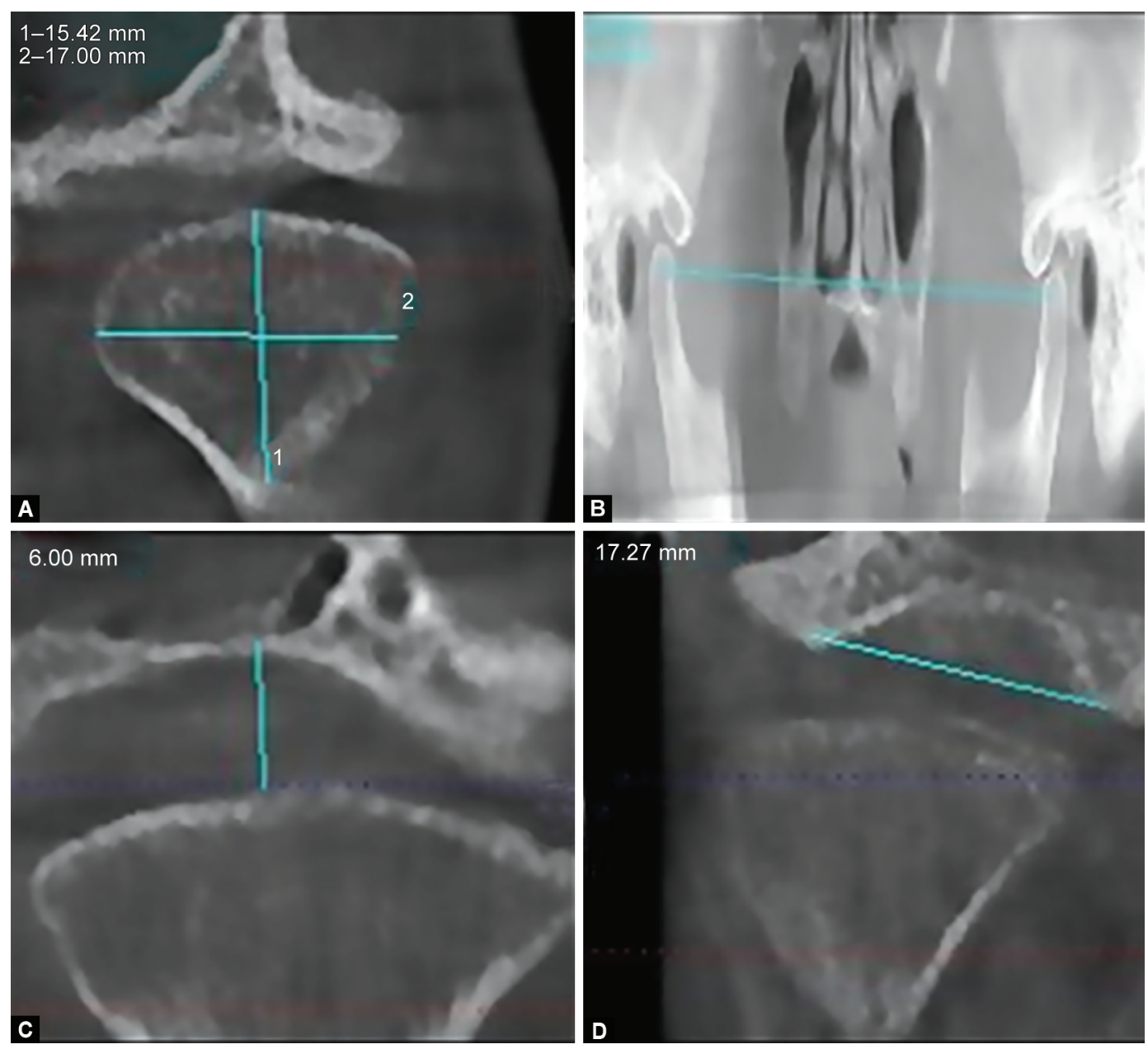

Figs 1 A to D: Analysis of CBCT images using an image processing tool: (A) Condyle distance measurement; (B) Interarticular distance measurement; (C) Glenoid fossa measurement; (D) Articular eminence measurement

Table 1: Intergroup comparison of condylar and interarticular distance $(\mathrm{mm})$ at different time periods

\begin{tabular}{|c|c|c|c|c|c|c|c|c|}
\hline \multirow[b]{3}{*}{ Time periods } & \multicolumn{4}{|c|}{ Condylar distance $(\mathrm{mm})$} & \multicolumn{4}{|c|}{ Interarticular distance $(\mathrm{mm})$} \\
\hline & \multirow{2}{*}{$\begin{array}{l}\text { Group I }(n=30) \\
\text { Mean } \pm S D\end{array}$} & \multirow{2}{*}{$\frac{\text { Group II }(n=30)}{\text { Mean } \pm S D}$} & \multicolumn{2}{|c|}{ Statistical significance } & \multirow{2}{*}{$\begin{array}{l}\text { Group I }(n=30) \\
\text { Mean } \pm S D\end{array}$} & \multirow{2}{*}{$\frac{\text { Group } / I(n=30)}{\text { Mean } \pm S D}$} & \multicolumn{2}{|c|}{ Statistical significance } \\
\hline & & & $' t$ & $p^{\prime}$ & & & ' $t$ ' & $p^{\prime}$ \\
\hline At insertion & $19.08 \pm 0.72$ & $19.22 \pm 0.66$ & -0.523 & $0.605^{*}$ & $101.83 \pm 4.54$ & $102.98 \pm 3.61$ & -0.770 & $0.448^{*}$ \\
\hline 6 m p.i. & $19.08 \pm 0.72$ & $19.22 \pm 0.66$ & -0.523 & $0.605^{*}$ & $101.83 \pm 4.54$ & $102.98 \pm 3.61$ & -0.770 & $0.448^{*}$ \\
\hline 12 m p.i. & $19.04 \pm 0.74$ & $19.21 \pm 0.66$ & -0.685 & $0.499^{*}$ & $101.81 \pm 4.54$ & $102.98 \pm 3.61$ & -0.779 & $0.443^{*}$ \\
\hline 18 m p.i. & $19.01 \pm 0.74$ & $19.21 \pm 0.66$ & -0.786 & $0.438^{*}$ & $101.80 \pm 4.54$ & $102.98 \pm 3.61$ & -0.784 & $0.439^{*}$ \\
\hline 24 m p.i. & $18.98 \pm 0.74$ & $19.20 \pm 0.66$ & -0.893 & $0.380^{*}$ & $101.77 \pm 4.55$ & $102.97 \pm 3.61$ & -0.806 & $0.427^{*}$ \\
\hline
\end{tabular}

${ }^{*} p$ value $>0.05$ is insignificant

was set at $p=0.05$. The statistical significance was derived using the one-sample $t$-test for intragroup and intergroup comparisons.

\section{Results}

Group I $(n=30)$ included randomly selected patients with mean age $59.00 \pm 6.74$ years. Whereas, group II $(n=30)$ included patients with mean age $58.27 \pm 6.75$ years. At insertion and after 6 months, mean intercondylar width in group I (19.08 $\pm 0.72 \mathrm{~mm})$ and group II was $19.22 \pm 0.66 \mathrm{~mm}$. At 12th, 18th, and 24th months, mean condylar distances were19.04 $\pm 0.74,19.01 \pm 0.72$, and $18.98 \pm 0.74$ $\mathrm{mm}$ in group I and for group II $19.21 \pm 0.66,19.21 \pm 0.66$ and 19.20 $\pm 0.66 \mathrm{~mm}$, respectively. In both the groups, no change in condylar distance was observed from baseline to 6 months post-insertion, but a continuous decline was observed with time (slight declination was noticed in both the groups (I and II) from baseline to 6 months post-insertion, which was not significant). At different periods, the level of significance for mean intercondylar width of both the groups was analyzed using the one-sample $t$-test and it was found to be statistically insignificant ( $p$ value $>0.05$ ) (Table 1).

In groups I and II, mean change in intercondylar width during $12-24$ months was $0.238-0.566 \%$ and $0.010-0.055 \%$, respectively. 
The one sample $t$-test was applied, and it was found that the level of significance is significant ( $p$ value $<0.05$ ) for both the groups, except for group II at 12 months post-insertion (Table 2).

At insertion and 6 months post-insertion, mean inter articular distance in patients of group II $(102.98 \pm 3.61 \mathrm{~mm})$ was higher than group I $(101.83 \pm 4.54 \mathrm{~mm})$ but was not statistically significant. The level of significance was analyzed using the one-sample $t$-test, and it was found that mean interarticular distances of groups I and II were not statistically significant at 12 months (101.81 \pm 4.54 and $102.98 \pm 3.61 \mathrm{~mm}), 18$ months $(101.80 \pm 4.54$ and $102.98 \pm$ $3.61 \mathrm{~mm})$, and 24 months $(101.77 \pm 4.55$ and $102.97 \pm 3.61 \mathrm{~mm})$ (Table 1). In group I, no change in mean interarticular distance was observed from baseline to 6 months, but decline at 12, 18, and 24 months was $0.013,0.024$, and $0.059 \%$, respectively, and was found to be statistically significant (Table 2). For group II, the percentage change in interarticular distance was significant at 24 months post-insertion only.

Mean length of glenoid fossa in group II was higher than group I at baseline, 6 months $(6.581 \pm 0.923$ and $6.125 \pm 1.272$ $\mathrm{mm}), 12$ months $(6.580 \pm 0.924$ and $6.104 \pm 1.271 \mathrm{~mm}), 18$ months $(6.577 \pm 0.922$ and $6.085 \pm 1.270 \mathrm{~mm})$, and 24 months $(6.575 \pm$ 0.920 and $6.060 \pm 1.269 \mathrm{~mm}$, respectively). Statistically, the level of significance was observed using the one-sample $t$-test and was found to be statistically insignificant at all periods of observation. A continuous decline in mean length of glenoid fossa in both groups was observed with time (Table 3 ).

In group I, change in length of glenoid fossa from baseline to 12 months was $0.348 \%, 0.664 \%$ at 18 months, and $1.067 \%$ at 24 months. Change in length was found to be statistically significant at 12,18 , and 24 months using the one-sample $t$-test statistical analysis. In group II, change in length of glenoid fossa from baseline to 12 months was $0.020 \%, 0.071 \%$ at 18 months, and $0.101 \%$ at 24 months. Statistically, it was found to be significant at 18 and 24 months for group II (Table 4).

At insertion and 6 months, mean articular eminence length of group II (18.559 $\pm 1.457 \mathrm{~mm}$ ) was higher than group I (18.375 \pm $1.990 \mathrm{~mm}$ ). Mean articular eminence length of group II was found to be higher than group I at 12 months $(18.559 \pm 1.457$ and $18.350 \pm$ $1.994 \mathrm{~mm}), 18$ months $(18.559 \pm 1.457$ and $18.319 \pm 1.995 \mathrm{~mm})$, and 24 months ( $18.559 \pm 1.457$ and $18.293 \pm 1.994 \mathrm{~mm}$ ), but differences were not found to be statistically significant (Table 3 ).

In group I, no change in articular eminence length was observed from its baseline to 6 months but a continuous decline was observed at 12 months ( $0.138 \%), 18$ months $(0.305 \%)$, and 24 months $(0.450 \%)$. Range of change in articular eminence length was found to be statistically significant $(p<0.05)$ using a one-sample $t$-test. In group II, no change was observed in articular eminence length from baseline to 12 months, but a decline was observed at 18 months $(0.002 \%)$ and 24 months (0.004\%). Change in the interarticular distance was found to be statistically significant only at 24 months (Table 4).

\section{Discussion}

According to the statistics, the elderly population of the civilized world has increased remarkably during the previous decades with various transformations that appear to represent an adaptation of condyle, articular disc, and articular eminence. The complete edentulousness over a long period may lead to several irreversible deformities like the anatomical changes of the TMJ.

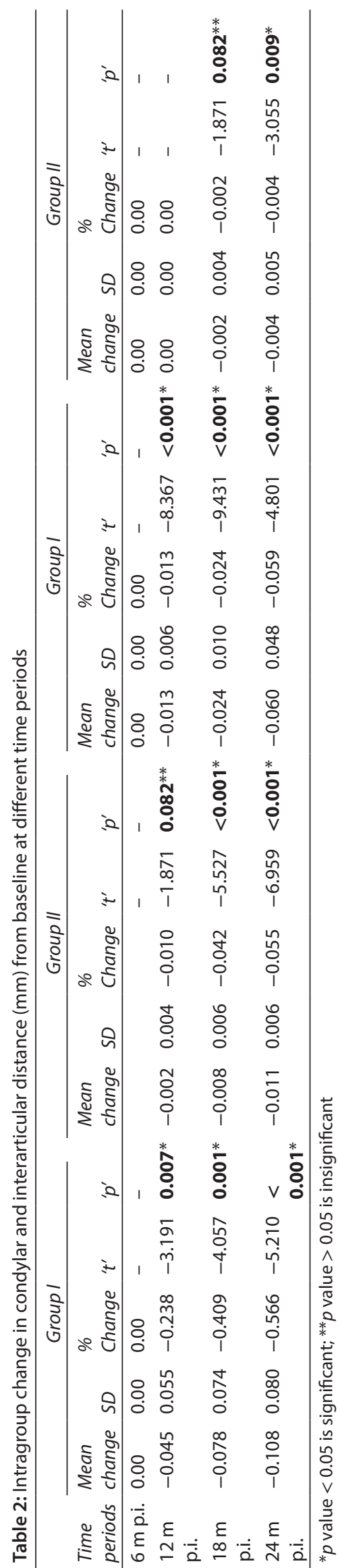



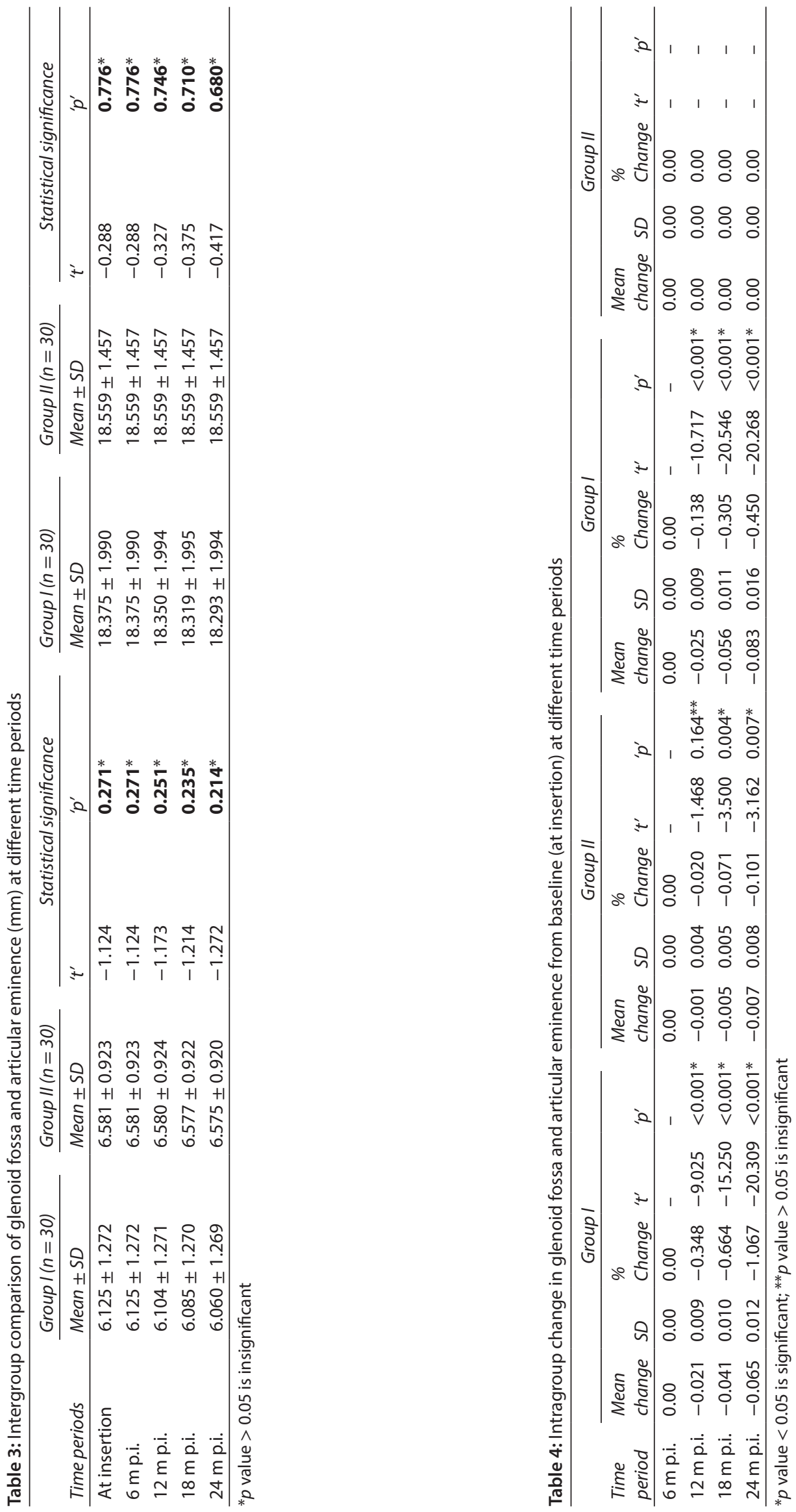
Several radiographic methods are used to assess degenerative bone changes that affect the TMJ. ${ }^{20}$ Multisliced CT represents an important advancement in this field that can reconstruct images with high resolution and less acquisition time. ${ }^{21}$ Recently, CBCT developed as an alternative to conventional CT, with good image quality, shorter patient examination time, and much lower radiation dose. Besides, the scanning procedure of the patient and the software for image reconstruction connected with CBCT are very user-friendly. ${ }^{8,22}$ Hence, the present study was conducted to evaluate the anatomical changes in the TMJ through CBCT in completely edentulous patients, after the insertion of the complete denture at the time interval of $6,12,18$, and 24 months, respectively.

Our analysis revealed that statistically there was no significant difference observed with varying time intervals of $6,12,18$, and 24 months for intercondylar width and interarticular distance in both the study groups. Results of the present study were as per the study conducted by Goiato et al., ${ }^{23}$ which showed that there were significant differences in the distribution of clinical symptoms related to the mandibular condyle and TMJ symptoms when an old denture is replaced by new pair of dentures.

In group II, glenoid fossa did not show any statistically significant changes as compared to group I, but a continuous decline in the mean length of glenoid fossa was observed in both the groups with time. Thus, changes in glenoid fossa were observed with time on wearing of dentures. The results of our study wherein as per the study conducted by Barghan et al. ${ }^{24}$ who also reported no significant correlation of the minimum thickness of the roof of the glenoid fossa (RGF) with gender and age. The results of our study were also in accordance with the study by Su et al. ${ }^{25}$ who concluded that sagittal osteoarthritic variations through mechanical stimulation and altered stress distribution might influence RGF thickness. Similar results were observed in our study, which showed changes in a glenoid fossa on variation in stress distribution on denture wearing.

A continuous decline in articular eminence length was observed for both the groups and change was found to be statistically significant. The results contrasted with the study conducted by Abdul-Nabi et al. ${ }^{26}$ who concluded that there were no statistically significant differences in articular eminence inclination and height. Wu et al. ${ }^{27}$ reported that there is no relation of articular eminence inclination with age, where eminence height and inclination have a significant effect by gender (favors result as the articular eminence shows significant changes regardless of gender).

The anatomical data obtained from the present study may be supportive to comprehend the variations in TMJ anatomy in edentulous patients depending on denture wearing, using CBCT. This study is one of the rare studies that analyzed the association of TMJ anatomy based on edentulism. Thus, further studies should be conducted on larger populations affecting the TMJ measurements. As observed in our study, we can conclude that there is a significant change in the parameters of TMJ in both the study groups, having varying degree of edentulism.

\section{Conclusion}

The present study concludes that the articular eminence flattening is correlated with age; on the other hand, the rate of deformation was found significantly more in total edentulous subjects as compared to subjects having normally maintained occlusion. The anatomical changes inside the temporomandibular joint have been much greater expressed within the completely edentulous subjects in whom the angle of sagittal condyle path declines and so does the articular eminence height.

\section{References}

1. Kijima N, Honda K, Kuroki Y, et al. Relationship between patient characteristics, mandibular head morphology and thickness of the roof of the glenoid fossa in symptomatic temporomandibular joints. Dentomaxillofac Radiol 2007;36(5):277-281. DOI: 10.1259/ dmfr/56344782.

2. Sümbüllü MA, Çağlayan F, Akgül HM, et al. Radiological examination of the articular eminence morphology using cone beam CT. Dentomaxillofac Radiol 2012;41(3):234-240. DOI: 10.1259/ $\mathrm{dmfr} / 24780643$.

3. Honey OB, Scarfe WC, Hilgers MJ, et al. Accuracy of cone-beam computed tomography imaging of the temporomandibular joint: comparisons with panoramic radiology and linear tomography. Am J Orthod Dentofacial Orthop 2007;132(4):429-438. DOI: 10.1016/j. ajodo.2005.10.032.

4. Shahab S, Nikkerdar N, Goodarzi M, et al. Diagnostic accuracy of cone beam computed tomography in detection of simulated mandibular condyle erosions. Dental Hypotheses 2015;6(3):97. DOI: 10.4103/21558213.163813.

5. Veloso L, Dias R, Messias A, et al. Evaluation of condylar position by CBCT after static and dynamic registration in edentulous patients. Revista Portuguesa de Estomatologia, MedicinaDentária e Cirurgia Maxilofacial 2015;56(1):9-17.

6. İlgüy $D$, İlgüy M, Fişekçioğlu $E$, et al. Articular eminence inclination, height, and condyle morphology on cone beam computed tomography. Sci World J 2014;2014:761714. DOI: 10.1155/2014/761714.

7. Krishnamoorthy B, Mamatha NS, Kumar VA. TMJ imaging by CBCT: current scenario. Ann Maxillofac Surg 2013;3(1):80. DOI: 10.4103/22310746.110069 .

8. Hintze $\mathrm{H}$, Wiese $\mathrm{M}$, Wenzel $\mathrm{A}$. Cone beam CT and conventional tomography for the detection of morphological temporomandibular joint changes. Dentomaxillofac Radiol 2007;36(4):192-197. DOI: $10.1259 / \mathrm{dmfr} / 25523853$.

9. Utumi ER, Perrella A, Albuquerque MA, et al. Evaluation of simulated bone lesion in the head of the mandible by using multislice computed tomography. J Appl Oral Sci 2009;17(5):521-526. DOI: 10.1590/S167877572009000500030.

10. deCóndilo ED, Detectado MB. Evaluation of the prevalence of bifid mandibular condyle detected on cone beam computed tomography images in a Turkish population. Int J Morphol 2015;33(1):43-47. DOI: 10.4067/S0717-95022015000100006.

11. Bastos LC, Campos PS, Ramos-Perez FM, et al. Evaluation of condyle defects using different reconstruction protocols of cone-beam computed tomography. Braz Oral Res 2013;27(6):503-509. DOI: 10.1590/S1806-83242013000600010.

12. Basili C, Costa HN, Sasaguri K, et al. Comparison of the position of the mandibular fossa using 3D CBCT in different skeletal frames in human caucasic skulls. Int J Stomatol Occlus Med 2009;2(4):179-190. DOI: 10.1007/s12548-009-0031-y.

13. Žabarović $D$, Jerolimov V, Carek V, et al. The effect of tooth loss on the TM-joint articular eminence inclination. Coll Antropol 2000;24(1):3742.

14. Ejima K, Schulze D, Stippig A, et al. Relationship between the thickness of the roof of glenoid fossa, condyle morphology and remaining teeth in asymptomatic European patients based on cone beam CT data sets. Dentomaxillofac Radiol 2013;42(3):90929410. DOI: 10.1259/ dmfr/90929410.

15. Zain-Alabdeen $\mathrm{EH}$, Alsadhan RI. A comparative study of accuracy of detection of surface osseous changes in the temporomandibular joint using multidetector CT and cone beam CT. Dentomaxillofac Radiol 2012;41(3):185-191. DOI: 10.1259/dmfr/24985971.

16. dos Anjos Pontual ML, Freire JS, Barbosa JM, et al. Evaluation of bone changes in the temporomandibular joint using cone beam CT. Dentomaxillofac Radiol 2012;41(1):24-29. DOI: 10.1259/dmfr/17815139. 
17. Al-Ekrish AA. A retrospective study of the prevalence and reliability of the diagnosis of soft tissue calcification of the temporomandibular joint in cone beam computed tomography images. King Saud Univers J Dent Sci 2013;4(2):81-85. DOI: 10.1016/j.ksujds.2013. 03.003.

18. Matsumoto K, Honda K, Sawada K, et al. The thickness of the roof of the glenoid fossa in the temporomandibular joint: relationship to the MRI findings. Dentomaxillofac Radiol 2006;35(5):357-364. DOI: $10.1259 / \mathrm{dmfr} / 30011413$.

19. Talaat W, Al Bayatti S, Al Kawas S. CBCT analysis of bony changes associated with temporomandibular disorders. CRANIO ${ }^{\circledR}$ 2016:34(2):88-94. DOI: 10.1179/2151090315Y.0000000002.

20. Westesson PL, Katzberg RW, Tallents RH, et al. CT and MR of the temporomandibular joint: comparison with autopsy specimens. AJR Am J Roentgenol 1987;148(6):1165-1171. DOI: 10.2214/ajr. 148.6.1165.

21. Scolozzi P, Momjian A, Courvoisier DS, et al. Evaluation of condylar morphology following orthognathic surgery on digital panoramic radiographs. Could methodology influence the range of "normality" in condylar changes? Dentomaxillofac Radiol 2013;42(7):20120463. DOI: 10.1259/dmfr.20120463.

22. Dallanora $A F$, Grasel $C E$, Heine $C P$, et al. Prevalence of temporomandibular disorders in a population of complete denture wearers. Gerodontology 2012;29(2):e865-e869. DOI: 10.1111/j.17412358.2011.00574.x.

23. Goiato MC, Rosalino Garcia A, Dos Santos DM, et al. TM vibrations in asymptomatic patients using old and new complete dentures. J Prosthodont 2010;19(6):438-442. DOI: 10.1111/j.1532849X.2010.00614.X.

24. Barghan S, Tetradis S, Mallya SM. Application of cone beam computed tomography for assessment of the temporomandibular joints. Aust Dent J 2012;57:109-118. DOI: 10.1111/j.1834-7819.2011.01663.x.

25. Su N, Liu Y, Yang X, et al. Correlation between bony changes measured with cone beam computed tomography and clinical dysfunction index in patients with temporomandibular joint osteoarthritis. J Craniomaxillofac Surg 2014;42(7):1402-1407. DOI: 10.1016/j. jcms.2014.04.001.

26. Abdul-Nabi LA, Al-Nakib LH. Flattening of the posterior slope of the articular eminence of completely edentulous patients compared to patients with maintained occlusion in relation to age using computed tomography. J Baghdad College Dentis 2015;325(2219):1-6. DOI: 10.12816/0015297.

27. Wu CK, Hsu JT, Shen YW, et al. Assessments of inclinations of the mandibular fossa by computed tomography in an Asian population. Clin Oral Investig 2012;16(2):443-450. DOI: 10.1007/s00784-0110518-y. 